'Servicio de Dermatología,

Hospital Clínico Universidad de Chile, Santiago, Chile.

¿Unidad de Trasplantes del

Hospital Clínico Universidad de Chile. Santiago, Chile.

${ }^{3}$ Servicio de Dermatología, Hospital Barros Luco. Santiago, Chile.

aBecada de Dermatología

${ }^{b} \mathrm{MSc}$ en Bioquímica.

'Enfermero universitario.

Conflicto de intereses: ninguno que declarar.

Fuente de apoyo financiero: sin apoyo externo.

Recibido el 19 de mayo de 2014, aceptado el 12 de marzo de 2015.

Correspondencia a: Francisca Daza Av. Vitacura 5951, Vitacura. Santiago, Chile. frandazap@gmail.com

\section{Manifestaciones cutáneas en adultos con trasplante hepático del Hospital Clínico de la Universidad de Chile}

\author{
FRANCISCA DAZA ${ }^{1, \mathrm{a}}$, JAIME PONIACHIK ${ }^{2}$, VIVIANA ZEMELMAN ${ }^{1, \mathrm{~b}}$, \\ JOSÉ IBARRA ${ }^{2, \mathrm{c}}$, MIGUEL ESPINOZA ${ }^{3}$, JAIME CASTILLO $^{2}$, \\ GONZALO CARDEMIL ${ }^{2}$, JUAN CARLOS DÍAZ ${ }^{2}$, ALEXANDRE SAURE ${ }^{2}$, \\ HANS LEMBACH ${ }^{2}$, PERLA CALDERÓN ${ }^{1}$
}

\section{Skin manifestations in adults with a liver allograft}

Background: Skin manifestations after liver transplantation are increasing due to long term immunosuppressive therapy along with an increase in patient survival. Several studies have reported dermatologic complications following renal transplant, but few have studied dermatologic problems after liver transplantation. Aims: To describe the different types of cutaneous lesions encountered in adults receiving a liver allograft. To evaluate the frequency of cutaneous manifestations of patients in the liver transplant waiting list. Material and Methods: Eighty patients submitted to a liver transplant and 70 patients in the liver transplant waiting list were evaluated with a complete dermatological physical examination. Results: Sixty one percent of patients with a liver allograft had at least one skin manifestation. Of these, $34 \%$ had superficial fungal infections, $31 \%$ had viral infections, 20\% had cutaneous side effects due to immunosuppressive treatment, 10\% had malignant lesions, $2 \%$ had bacterial infections and one patient had a graft versus host disease. Only $28 \%$ of patients in the liver transplant waiting list had dermatologic problems, and the vast majority were lesions linked to liver cirrhosis. Conclusions: Cutaneous infections were the most common skin problems in liver transplant patients. Although neoplastic lesions are the most commonly mentioned lesions in the literature, only a $10 \%$ of our liver transplant patients presented these type of lesions.

(Rev Med Chile 2015; 143: 584-588)

Key words: Chile; Liver transplantation; Skin Manifestations.
$\mathrm{E}$ 1 número de trasplantes de órgano sólido ha aumentado a nivel mundial y en Chile. El primer trasplante hepático ortotópico (THO) se realizó en Denver, USA, el año $1963^{1}$. Actualmente, el trasplante hepático ortotópico es a menudo la opción terapéutica final tanto en enfermedades hepáticas crónicas, como en falla hepática fulminante. El THO tiene por objetivo prolongar la sobrevida del paciente con una buena calidad de vida ${ }^{2}$. La inmunosupresión crónica, necesaria para evitar el rechazo del órgano tras- plantado, aumenta el riesgo de complicaciones dermatológicas ${ }^{3}$. Las causas más frecuentes de dermatosis en el paciente trasplantado se agrupan según su etiopatogenia en infecciones, neoplasias, reacciones adversas a medicamentos y otras secundarias al trasplante como la enfermedad injerto versus huésped $(\mathrm{EICH})^{4-8}$.

Entre los trasplantes de órganos sólidos, el trasplante de hígado es el que requiere menor grado de inmunosupresión ${ }^{9}$, por esta razón, los pacientes sometidos a trasplante de hígado pre- 
sentarían un menor número de complicaciones dermatológicas que los recipientes de otros órganos sólidos ${ }^{9-13}$. Igualmente, estudios descriptivos internacionales describen un riesgo aumentado de infecciones cutáneas, cáncer de piel y reacciones adversas a medicamentos en pacientes con $\mathrm{THO}^{14-}$ ${ }^{19}$. En Chile, no hay estudios publicados sobre las lesiones cutáneas encontradas en pacientes con trasplante de hígado.

Los objetivos de este trabajo son describir y determinar los diferentes tipos de manifestaciones dermatológicas encontradas en pacientes adultos con trasplante hepático en el Hospital Clínico de la Universidad de Chile (HCUCH) y comparar la frecuencia de enfermedades dermatológicas con los pacientes en lista de espera para THO.

\section{Pacientes y Método}

Se realizó un estudio observacional, descriptivo y exploratorio.

\section{Pacientes:}

Grupo de estudio: De los 160 pacientes que sufrieron trasplante de hígado en el Hospital Clínico de la Universidad de Chile (HCUCH) desde agosto del año 2002 hasta octubre del año 2011, 80 pacientes fueron evaluados.

Grupo control: Pacientes con cirrosis hepática en lista de espera del HCUCH.

\section{Metodología}

Se citó a los pacientes a un control ambulatorio y se recopilaron los datos relevantes para el estudio. Se realizó un examen físico dermatológico que incluyó: piel, fototipo de piel, mucosas y fanéreos. El fototipo de piel fue registrado según Fitzpatrick (I, II, III, IV, V, VI). A toda lesión sospechosa de malignidad o con diagnóstico incierto se le realizó una biopsia de piel para confirmar el diagnóstico.

Los criterios de inclusión utilizados para el estudio fueron: pacientes mayores de 15 años, pacientes con trasplante de hígado o en lista de espera para ser trasplantados en el HCUCH. Los pacientes debían aceptar ingreso al estudio mediante un consentimiento informado.

Se realizó una caracterización general de ambos grupos mediante un análisis descriptivo. Para estudiar las manifestaciones cutáneas en los dos grupos se realizó una comparación análisis multivariable donde la variable independiente fue la presencia o ausencia de tratamiento inmunosupresor y las variables dependientes fueron las manifestaciones cutáneas. El nivel de significancia estadística escogido fue $\mathrm{p}<0,01$.

\section{Resultados}

Se incluyeron 80 pacientes con trasplante hepático del HCUCH para el grupo de estudio y a 70 pacientes en lista de espera para trasplante hepático como grupo control.

\section{Caracterización del grupo de THO}

De 80 pacientes, $41(51 \%)$ eran de sexo femenino.

El promedio de edad de los pacientes al momento de ser examinados fue de 55,8 $\pm 11,4$ años. En cuanto al fototipo de piel según Fitzpatrick, 12 pacientes $(15 \%)$ presentaron un fototipo II, 41 pacientes $(51 \%)$ presentaron un fototipo III y 27 pacientes presentaron un fototipo IV (34\%). Ninguno de los pacientes presentó el antecedente familiar ni personal de cáncer de piel.

El promedio de edad de los pacientes al momento del trasplante fue de $52,5 \pm 12$ años, con un rango de edad entre 13 y 69 años. Se detectó que la edad promedio al momento del trasplante era mayor en varones (55,9 años) que en mujeres (49 años), $\mathrm{p}<0,01$. La principal causa de cirrosis en el grupo de trasplantados fue cirrosis por virus hepatitis C $(18,8 \%)$ y la principal causa de cirrosis en el grupo control fue esteatohepatitis no alcoholica $(41,4 \%)$. El tiempo promedio transcurrido desde el trasplante al corte transversal fue de 3,7 $\pm 2,6$ años, con un rango de 3 meses a 9,25 años. En relación a los fármacos inmunosupresores, 55 pacientes utilizaron tacrólimus $(68,75 \%), 22$ pacientes utilizaron ciclosporina $(27,5 \%), 15$ utilizaron mofetil micofenolato $(18,75 \%)$ y 4 utilizaron rapamicina (5\%). En el momento del estudio 22 pacientes estaban además con prednisona $(27,5 \%)$. De los 80 pacientes trasplantados, $49(61,25 \%)$ presentaron alguna manifestación dermatológica después de haber recibido un trasplante hepático. Se registraron un total de 61 patologías dermatológicas que se clasificaron en diferentes grupos según etiología (Figura 1). Diez pacientes presentaron más de un tipo de manifestación dermatológica. En conjunto, las infecciones cutáneas constituyen el grupo más 


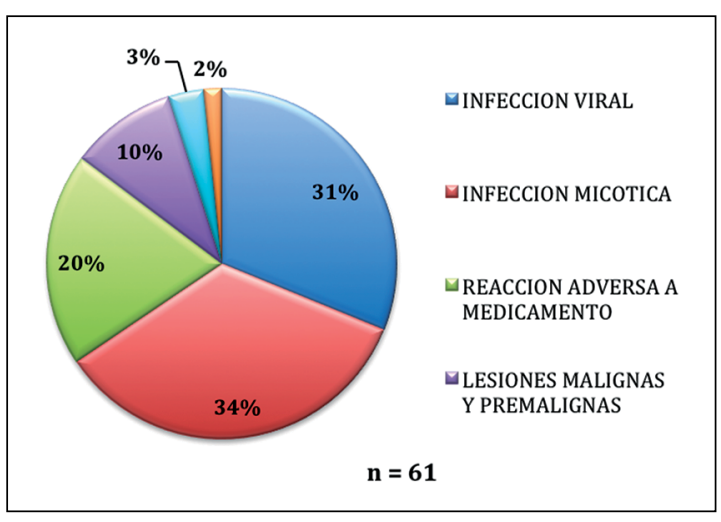

Figura 1. Manifestaciones dermatológicas en pacientes con trasplante hepático.

frecuente, representando el $71 \%$ del total de las dermatosis encontradas en los pacientes con THO. Dentro de estas infecciones cutáneas, las infecciones micóticas superficiales (34\%) y las infecciones virales (31\%) fueron las más frecuentes (Figura 1). El $20 \%$ de las dermatosis encontradas fueron reacciones adversas a medicamentos (RAM). La RAM más frecuente fue la hipertrocosis asociada a ciclosporina. De los 80 pacientes con THO, $7,5 \%$ presentó alguna lesión maligna después del trasplante, constituyendo $10 \%$ del total de dermatosis encontradas. Todos los pacientes de este grupo presentaron un fototipo de piel tipo II según Fitzpatrick. Se detectó un paciente de sexo masculino, de 63 años que tenía el antecedente de haber presentado hacía 5 años enfermedad injerto contra huésped (EICH).

\section{Caracterización del grupo control}

Del total de pacientes en lista de espera para trasplante hepático, 38 (54\%) eran de sexo masculino.

El promedio de edad de los pacientes estudiados fue de $60 \pm 8,2$ años. Con rango de 36 a 71 años. En cuanto al fototipo de piel según Fitzpatrick, 6 pacientes $(9 \%)$ presentaron un fototipo II, 41 pacientes $(59 \%)$ presentó un fototipo III y 23 pacientes $(33 \%)$ presentaron un fototipo IV. Ninguno de los pacientes pretrasplante presentó el antecedente familiar ni personal de cáncer de piel. Todos los pacientes referían haber tenido una fotoexposición normal.

La principal causa de cirrosis en este grupo fue la esteatohepatitis no alcohólica (NASH).
De los 70 pacientes analizados, $20(28,6 \%)$ pacientes presentaron algún tipo de manifestación dermatológica. Dentro de las manifestaciones dermatológicas observadas, se encontró $70 \%$ de signos cutáneos asociados a daño hepático crónico y $30 \%$ correspondía a infecciones micóticas.

\section{Comparación de las manifestaciones cutáneas en pacientes con $y \sin \mathrm{THO}$}

En el grupo control se observaron signos cutáneos propios de las hepatopatías crónicas en $70 \%$ de los pacientes, en cambio en los pacientes trasplantados no se observaron manifestaciones cutáneas por efecto de hepatopatías crónicas.

Los pacientes con THO presentaron mayor frecuencia de enfermedades dermatológicas que el grupo control. El porcentaje de pacientes con manifestaciones dermatológicas en el grupo con THO fue de $61,25 \%$, en cambio, el porcentaje de pacientes con manifestaciones dermatológicas en el grupo control fue de 28,75\% ( $\mathrm{p}<0,01)$.

Cuando se comparó por tipo específico de patología dermatológica, se observó que el grupo de estudio tiene un mayor porcentaje de infecciones micóticas, de infecciones virales, de queratosis actínicas y de hipertricosis que el grupo control. Estas diferencias fueron estadísticamente significativas $(\mathrm{p}<0,05)($ Tabla 1$)$.

\section{Discusión}

El presente estudio mostró que la prevalencia de enfermedades dermatológicas en pacientes con THO fue de $61 \%$. El promedio de tiempo de presentación después del trasplante fue de 3,4 \pm 2,1 años, este período es similar al reportado por otros estudios internacionales ${ }^{18-19}$.

Dentro de las manifestaciones dermatológicas, las más frecuentes fueron las infecciones micóticas (34\%) al igual que un estudio descriptivo realizado en Inglaterra con 100 pacientes a los 5 años post trasplante ${ }^{18}$, que reportó una mayor frecuencia de infecciones micóticas (19\%). El segundo grupo en frecuencia en nuestro estudio fueron las infecciones virales, que representaron $31 \%$ de las manifestaciones dermatológicas encontradas. Cabe destacar que todos los casos de herpes simple, al igual que de herpes zoster, ocurrieron dentro del primer año post trasplante.

Se describe en la literatura que en el trasplante 
Tabla 1. Comparación de las manifestaciones dermatológicas entre el grupo de estudio y el grupo control

\begin{tabular}{|c|c|c|c|c|c|}
\hline \multirow{2}{*}{$\begin{array}{l}\text { Manifestación } \\
\text { dermatológica }\end{array}$} & \multicolumn{2}{|c|}{ Grupo estudio } & \multicolumn{2}{|c|}{ Grupo control } & \multirow{2}{*}{$\begin{array}{l}\text { Valor } \\
\text { de p }\end{array}$} \\
\hline & $\begin{array}{l}\text { n de pacientes } \\
\text { comprometidos }\end{array}$ & $\begin{array}{l}\% \text { de pacientes } \\
\text { comprometidos }\end{array}$ & $\begin{array}{l}\text { n de pacientes } \\
\text { comprometidos }\end{array}$ & $\begin{array}{l}\% \text { de pacientes } \\
\text { comprometidos }\end{array}$ & \\
\hline Infecciones micóticas & 21 & 26,25 & 6 & 8,57 & $p<0,01$ \\
\hline Onicomicosis & 19 & 23,75 & 4 & 5,71 & $p<0,01$ \\
\hline Infecciones virales & 19 & 23,75 & 0 & 0 & $p<0,01$ \\
\hline Verrugas & 10 & 12,50 & 0 & 0 & $p<0,01$ \\
\hline Hipertricosis (RAM) & 12 & 15 & 0 & 0 & $p<0,01$ \\
\hline Queratosis actínicas & 6 & 7,50 & 0 & 0 & $p=0,03$ \\
\hline Lesiones malignas y premalignas & 6 & 7,50 & 0 & 0 & $p=0,03$ \\
\hline Sin lesiones cutáneas & 31 & 38,75 & 50 & 71,43 & $p<0,01$ \\
\hline
\end{tabular}

hepático, la frecuencia de lesiones malignas es menor que en los pacientes con trasplante renal ${ }^{15}$. En esta investigación, sólo 6 pacientes presentaron Queratosis actínicas (QA) y uno de ellos presentó además 2 Carcinomas basocelulares (CBC). El porcentaje de lesiones cutáneas pre malignas y malignas observado en nuestro trabajo (10\%) es menor que lo reportado internacionalmente por Perera et $\mathrm{al}^{18}$ a 5,5 años de seguimiento y lo reportado por Belloni-Fortina et $\mathrm{al}^{20}$ a 6 años de seguimiento. Estas diferencias se podrían deber a múltiples causas, una de ellas al fenotipo de piel más oscuro de nuestros pacientes, de hecho $85 \%$ de los trasplantados tenían fototipo III y IV. Los 6 casos descritos anteriormente tenían un fototipo II según Fitzpatrick. También, podría deberse a que un gran porcentaje de nuestros pacientes usaban Tacrolimus como droga inmunosupresora y se ha descrito que cuando se usa un solo tratamiento inmunosupresor, la inmunosupresión es menor que cuando se usa una doble o triple terapia inmunosupresora ${ }^{21}$. En nuestros pacientes, más de la mitad de los pacientes tratados con ciclosporina presentaron hipertricosis, este fenómeno es una reacción adversa conocida de la ciclosporina, y se ha descrito hasta en $90 \%$ de los usuarios ${ }^{22}$.

Se ha observado que la enfermedad de injerto contra huésped (EICH) es más frecuente en trasplante hepático que en otros trasplantes de órganos sólidos ${ }^{10-13}$, en estos pacientes presenta una incidencia de 0,1 a $1 \%{ }^{10-12}$. En nuestro estudio, sólo un paciente tenía el antecedente de haber presenta- do un EICH cutáneo a los 6 meses post trasplante. El paciente presentó un rash liquenoide generalizado como primer signo clínico, coincidiendo con lo descrito en la literatura internacional ${ }^{10,12-13}$.

En nuestro estudio, no se observaron signos clínicos cutáneos por efecto del daño hepático crónico (DHC) en los pacientes trasplantados. Esto confirma hallazgos realizados por otros investigadores que observan que los signos clínicos cutáneos del daño hepático crónico (DHC) se resuelven después de realizado el trasplante hepático ${ }^{14-19}$.

Los pacientes del grupo control, en lista de espera para THO, presentaron características similares al grupo de estudio en cuanto a sexo, edad. La patología dermatológica encontrada en estos pacientes fue diferente al grupo de pacientes trasplantados. En los pacientes del grupo control, las manifestaciones cutáneas más frecuentes fueron los signos dermatológicos asociados a daño hepático crónico. En el grupo control, 14,28\% presentó infecciones micóticas, pero aún así la frecuencia fue significativamente menor que en los pacientes con THO.

Una de las principales limitaciones de este estudio es que se obtiene únicamente una medición de las exposiciones y eventos en los sujetos de estudio en un momento dado; debido a esto, no es posible determinar si el supuesto factor de exposición precedió al aparente efecto. Con el grupo adicional elegido se logra atenuar esta limitación y deducir causalidad entre exposición y efecto. 
En conclusión, este estudio muestra que los pacientes con trasplante hepático tienen un alto índice de manifestaciones cutáneas. Las principales enfermedades dermatológicas encontradas fueron infecciones micóticas, infecciones virales, hipertricosis asociada a ciclosporina y lesiones pre-malignas y malignas. Este es el primer estudio realizado en Chile sobre las manifestaciones dermatológicas en trasplante hepático. Los datos obtenidos sirven para determinar la importancia de prevenir y tratar tempranamente los trastornos dermatológicos más frecuentes. Además, se hace indispensable la necesidad de colaboración entre hepatólogos y dermatólogos en la educación y seguimiento de los pacientes con trasplante hepático.

\section{Referencias}

1. Starzl TE, Marchioro TL, Von Kaulla KN, Hermann G, Brittain RS, Wad-dell WR. Homo transplantation of the liver in humans. Surg Gynecol Obstet 1963; 117 (1): 659-76.

2. Hepp J, Zaror M, Ríos H, Suárez L, Figueroa P, Quiroga M, et al. Perspectivas del Trasplante Hepático en Chile. Rev Med Chile 1990; 118 (4): 453-8.

3. Otley CC, Stasko T. Skin Disease in Organ Transplantation. $1^{\text {st }}$ edition. New York: Cambridge University Press, 2008. p341. Foreword, 3-9.

4. Otley CC, Stasko T. Skin Disease in Organ Transplantation. $1^{\text {st }}$ edition. New York: Cambridge University Press, 2008. p341. Chapters 4-7, 60-141.

5. Glover MT, Deeks JJ, Raftery MJ, Cunningham J, Leigh IM. Immunosuppression and risk of non-melanoma skin cancer in renal transplant recipients. Lancet 1997; 349 (9049): 398.

6. Hogewoning AA, Goettsh W, van Loveren H, de Fijter JW, Vermeer BJ, Bouwes Bavinck JN. Skin infections in renal transplant patients. Clin Transplan 2001; 15 (1): 32-8.

7. Savoia P, Stroppiana E, Cavaliere G, Osella-Abate S, Mezza E, Segoloni GP, et al. Skin cancers and other cutaneous diseases in renal transplant recipients: a single Italian center observational study. Eur J Dermatol 2011; 21 (2): 242-7.

8. Dufrechou L, Borges AL, Nin M, et al. Cutaneous manifestations in 100 renal and renopancreatic recipients of Uruguay. Transplant Proc 2011; 43 (9): 3377-9.

9. Ramos HC, Reyes J, Abu-Elmagd K, Curi L, González
F, Martínez M, et al. Weaning of immunosupresion in long-term liver transplant recipients. Transplantation 1995; 59 (2): 212-7.

10. Roberts JP, Ascher NL, Lake J, Capper J, Purohit S, Garovoy M, et al. Graft vs host disease after liver transplantation in humans: a report of four cases. Hepatol 1991; 14 (2): 274-81.

11. Redondo P, España A, Herrero JI, Quiroga J, Cienfuegos JA, Azanza JR, et al. Graft-versus-host disease after liver transplantation. J Am Acad Dermatol 1993; 29 (2 Pt 2): 314-7.

12. Smith DM, Agura E, Netto G, Collins R, Levy M, Goldstein R, et al. Liver transplant-associated graftversus-host disease. Transpl 2003; 75 (1): 118-26.

13. Kohler S, Pascher A, Junge G, Sauer IM, Nagy M, Schönemann C, et al. Graft versus host disease after liver transplantation-a single center experience and review of literature. Transpl 2008; 21 (5): 441-51.

14. Reggiani M, Pauluzzi P, Bellusci R, De Raffele E, Nardo B, Gozzetti G. Dermatologic problems in patients undergoing liver transplantation. G Ital Dermatol Venereol 1989; 124: (9) 393-6.

15. Otley CC, Pittelkow MR. Skin cancer in liver transplant recipients. Liver Transpl 2000; 6 (3): 253-62.

16. Sánchez EQ, Marubashi S, Jung G, Levy MF, Goldstein RM, Molmenti EP, et al. De novo tumors after liver transplantation: A single-institution experience. Liver Transpl 2002; 8 (3): 285-91.

17. Schmied E, Dufour JF, Euvrard S. Nontumoral dermatologic problems after liver transplantation. Liver Transpl 2004; 10 (3): 331-9.

18. Perera GK, Child FJ, Heaton N, O'Grady J, Higgins EM. Skin lesions in adult liver transplant recipients: a study of 100 consecutive patients. Br J Dermatol 2006; 154 (5): 868 -

19. Hassan G, Khalaf H, Mourad W. Dermatologic complications after liver transplantation: a single-center experience. Transplant Proc 2007; 39 (4): 1190-4.

20. Belloni-Fortina A, Piaserico S, Bordignon M, Gambato M, Senzolo M, Russo FP, et al. Skin cancer and other cutaneous disorders in liver transplant recipients. Acta Derm Venereol 2012; 92 (4): 411-5.

21. Jensen P, Møller B, Hansen S, Leivestad T, Pfeffer P, Geiran O, et al. Skin cancer in kidney and heart transplant recipients and different long-term immunosuppressive therapy regimens. J Am Acad Dermatol 1999; 40 (2 Pt 1): 177-86.

22. Wysocki G, Daley T. Hypertrichosis in patients receiving cyclosporine therapy. Clin Exp Dermatol 1987; 12 (3): 191-6. 